\title{
Desigualdades geográficas e sociais na utilização de serviços de saúde no Brasil ${ }^{\star}$
}

\author{
Social and geographical inequalities \\ in health services utilization in Brazil
}

Cláudia Travassos 1

Francisco Viacava 1

Cristiano Fernandes 2

Célia Maria Almeida 2
1 Departamento

de Informações

em Saúde, Centro

de Informação Científica

e Tecnológica (Cict),

Fundação Oswaldo Cruz.

Av. Brasil 4.365, 21045-900

Rio de Janeiro, RJ.

claudia@malaria.procc.

fiocruz.br

2 Departamento

de Administração e

Planejamento em Saúde,

Escola Nacional de Saúde

Pública (Ensp), Fundação

Oswaldo Cruz.
Abstract Health care service consumption is related not only to need and individuals' behavior, but also to factors associated to the supply side of health care market. The new Brazilian Constitution (1988) established the Unified Health Care System (SUS) which is based on universal access to health care services. The system was implemented in 1990. The principle of equity can be identified in the health sector legislation and can be translated as equal opportunity of access to equal needs. This study aimed at evaluating equity in the use of health care services considering two dimensions: geographical and social dimensions. Data came from two general household surveys carried out in 1989 and 1996/1997 by the Brazilian Census Bureau (IBGE). Standardized utilization rates controlled by morbidity and insurance coverage were used to analyze geographical variation. Private expenditure with health insurance and drugs was also compared. Logistic regression was used to test for social inequalities. Results of the study suggest small reduction in inequalities between 1989 and 1996/1997, indicating that Brazilian health care system remains highly unequal.

Key words Equity; Health Care Service Consumption; Health Policy; Health Care Service Utilization; Private Expenditure on Health
Resumo O consumo de serviços de saúde é função das necessidades e do comportamento dos indivíduos em relação a seus problemas de saúde, bem como das formas de financiamento e dos serviços e recursos disponíveis para a população. A Constituição brasileira de 1988 estabelece o Sistema Único de Saúde (SUS) com base na institucionalização da universalidade da cobertura e do atendimento. O sistema foi implementado em 1990 e pode ser traduzido como igualdade de oportunidade de acesso aos serviços de saúde para necessidades iguais. Este trabalho estuda a eqüidade no uso de serviços de saúde a partir de duas dimensões: a geográfica e a social. Os dados utilizados são de pesquisas realizadas em 1989 e 1996-1997, pelo IBGE. Para avaliar as desigualdades geográficas no consumo de serviços de saúde foram calculadas taxas padronizadas de utilização de serviços. Comparou-se também a dimensão do gasto privado domiciliar com medicamentos e com planos de saúde. Para avaliar as desigualdades sociais, estimou-se a razão de odds para três grupos de renda e para as pessoas com e sem cobertura de plano de saúde. Observou-se pequena redução dos níveis de desigualdades no período analisado (1989-1996/1997), com o sistema de saúde atual mantendo-se caracterizado por marcadas iniqüidades.

Palavras-chave Eqüidade; Consumo de Serviços de Saúde; Política de Saúde; Utilização de Serviços de Saúde; Gasto Privado em Saúde 


\section{Introdução}

O padrão de utilização de serviços de saúde de um determinado grupo populacional é principalmente explicado por seu perfil de necessidades em saúde (Hulka \& Wheat, 1985). Está condicionado, também, por inúmeros outros fatores, internos e externos ao setor, relacionados tanto à forma como está estruturada a oferta de serviços (Wennberg, 1985) quanto às preferências e escolhas do usuário.

A disponibilidade, o tipo, a quantidade de serviços e recursos (financeiros, humanos, tecnológicos), a localização geográfica, a cultura médica local, a ideologia do prestador, entre outros, são aspectos da oferta que influenciam o padrão de consumo de serviços de saúde. Por outro lado, as escolhas individuais também são cruciais, embora nem todas as necessidades se convertam em demandas e nem todas as demandas sejam atendidas. Inversamente, por indução da oferta, existe o uso de serviços não relacionados com as necessidades. $\mathrm{Na}$ verdade, segundo a Lei de Hart (1971), os diversos mecanismos que interferem na oferta de serviços fazem com que os recursos sejam distribuídos inversamente às necessidades.

Assim, desigualdades no uso de serviços de saúde, isto é, na atitude de procurá-los, obter acesso e se beneficiar com o atendimento recebido, refletem as desigualdades individuais no risco de adoecer e morrer, assim como as diferenças no comportamento do indivíduo perante a doença, além das características da oferta de serviços que cada sociedade disponibiliza para seus membros.

Em outras palavras, o uso de serviços de saúde é função das necessidades e do comportamento dos indivíduos diante dos seus problemas de saúde, assim como das formas de financiamento, dos serviços e recursos disponíveis para a população, incluindo a estrutura administrativa e os mecanismos de pagamento.

Embora as noções de igualdade e eqüidade sejam utilizadas indistintamente, políticas voltadas para eqüidade pressupõem redistribuição desigual de recursos, por causa dos ajustes que devem ser efetuados em função de fatores biológicos, sociais e político-organizacionais determinantes das desigualdades existentes (Porto, 1997; Almeida et al., 1999a).

A questão da eqüidade em saúde tem sido objeto de intenso debate nas últimas décadas, $e$ as dificuldades encontradas para a sua con- ceituação resultam também do insuficiente desenvolvimento teórico que lhe serve de sustento (Porto, 1997). Algumas das questões referentes a esta temática são recorrentes, como as derivadas das relações entre condições socioeconômicas e saúde (Illsley \& Wnuk-Lipinski, 1990; Illsley \& Baker, 1991; Castellanos, 1997; Black et al. 1990); bem como aquelas voltadas à articulação entre desigualdade em saúde e desigualdade no acesso a bens e serviços (Daniels, 1982; Whitehead, 1992; Carr-Hill, 1994; Travassos, 1992).

Faz-se necessário também distinguir eqüidade em saúde de eqüidade no uso ou no consumo de serviços de saúde, porque os determinantes das desigualdades no adoecer e no morrer diferem daqueles das desigualdades no consumo de serviços de saúde. As desigualdades em saúde refletem, dominantemente, as desigualdades sociais e, em função da relativa efetividade das ações de saúde, a igualdade no uso de serviços de saúde é condição importante, porém, não suficiente para diminuir as desigualdades no adoecer e morrer (Travassos, 1992).

Vários estudos indicam que a posição do indivíduo na estrutura social é importante preditor de necessidades em saúde, e o padrão de risco observado tende a ser desvantajoso para aqueles indivíduos pertencentes aos grupos sociais menos privilegiados (Evans, 1994). No que toca à necessidade, não existe uma definição única, nem uma metodologia única para medi-la. Em geral, os autores afirmam que, quaisquer que sejam as formas de aferição, sempre expressarão visões parciais, limitadas, tanto por problemas metodológicos, quanto por percepções e valores próprios, seja do indivíduo, seja do avaliador. Existem também dificuldades importantes com relação aos conceitos e às formas de operacionalização das variáveis referentes à condição social dos indivíduos.

A questão da eqüidade tem sido enfatizada a partir das décadas de 1970-1980, quando a discussão da crise do Estado de Bem-Estar Social suscitou o questionamento dos resultados em termos de benefícios dos investimentos realizados nos sistemas de saúde (a extensa intervenção estatal, isto é, o montante do financiamento público, a provisão estatal de serviços etc.). Este tema ressurge nas discussões sobre as reformas setoriais, que colocam em pauta a necessidade de produção de estudos que avaliem o desempenho das diferentes 
formas de organização de serviços na superação das desigualdades no setor. De maneira geral, pode-se afirmar que persistem profundas incertezas com relação ao impacto dessas reformas sobre a eqüidade (Almeida et al., 1999a).

O sistema de saúde brasileiro é constituído por uma complexa rede de prestadores e compradores de serviços, simultaneamente interrelacionados, complementares e competitivos, formando um complicado mix público e privado, financiado majoritariamente com recursos públicos. Compõe-se por três principais subsetores: 1) o público, com serviços financiados e prestados pelo Estado, nos seus diversos níveis, incluindo-se os serviços próprios das forças armadas; 2 ) o privado (lucrativo e não-lucrativo), financiado por sistemas de reembolso, que podem ser recursos públicos ou privados; 3 ) o de seguros privados, financiados diretamente pelo consumidor ou pelas empresas empregadoras (em geral de forma parcial), com diferentes níveis de preços e subsídios. A pluralidade do sistema de saúde brasileiro caracteriza-se, portanto, pela presença de diversos mercados de serviços de saúde com implicações variadas sobre a eqüidade no consumo. Além disso, é um sistema altamente centrado na assistência médica e no atendimento hospitalar, características que se acentuaram a partir dos anos 60 com o desenvolvimento da seguridade social.

Com a crise econômica na década de 1980, reduziram-se significativamente as receitas fiscais e contribuições sociais e houve forte impacto no volume de recursos destinados à assistência à saúde, cuja cobertura na época estava praticamente universalizada (Travassos, 1992).

A Constituição de 1988 introduz importantes modificações no sistema de saúde do país com a criação do Sistema Único de Saúde (SUS), implementado a partir de 1990, institucionalizando a universalidade da cobertura e do atendimento, assim como a uniformidade e equivalência dos benefícios e serviços de saúde para populações urbanas e rurais. Da carta constitucional e da legislação específica infere-se que o princípio da eqüidade pode ser traduzido como igualdade de oportunidade de acesso aos serviços de saúde para necessidades iguais.

Ainda são escassos os estudos voltados para mensurar o impacto da implantação do SUS sobre a eqüidade. Isto pode ser explicado, em parte, pelo fato de que dados sobre acesso e utilização de serviços de saúde, gerados a partir de inquéritos populacionais de âmbito nacional, são anteriores à implantação do SUS, como aqueles produzidos pelos suplementos saúde da Pesquisa Nacional por Amostra de Domicílios (PNAD), de 1981 e 1986, e pela Pesquisa Nacional sobre Saúde e Nutrição (PNSN) de 1989. Atualmente, embora restritos às regiões Nordeste e Sudeste, dados da Pesquisa sobre Padrões de Vida (PPV), de 1996/ 1997, possibilitam o estudo do padrão de eqüidade após a implantação do SUS.

Neste trabalho, o principal foco de atenção está no estudo da eqüidade no uso de serviços de saúde, analisada a partir de duas dimensões: a geográfica e a social (Travassos, 1997). A dimensão geográfica diz respeito às variações entre áreas, a dimensão social, às variações entre grupos sociais intra-áreas. Em um contexto de abrangência nacional, essas duas dimensões são complementares, na medida que uma política de eqüidade na dimensão social pressupõe uma distribuição eqüitativa de recursos entre áreas. Entretanto, embora necessária, a eqüidade geográfica não é condição suficiente para o alcance da eqüidade social.

O objetivo deste artigo é avaliar o padrão de eqüidade no consumo de serviços de saúde em dois momentos, antes da criação do SUS e depois da sua implantação. A análise centrou-se nas regiões Nordeste e Sudeste e utilizou os dados disponíveis em 1989 e 1996/1997. Buscou-se medir a ocorrência de mudança nas dimensões da eqüidade geográfica e social, em cada uma das regiões estudadas.

\section{Metodologia}

Os dados utilizados são oriundos da Pesquisa Nacional sobre Saúde e Nutrição (PNSN), de 1989, e da Pesquisa sobre Padrões de Vida (PPV), de 1996/97, ambas realizadas pela Fundação Instituto Brasileiro de Geografia e Estatística (IBGE).

A PNSN foi o último inquérito populacional de nível nacional realizado no país, que investigou, de forma relativamente ampla, aspectos referentes à morbidade e à utilização de serviços de saúde. A amostra da PNSN é estratificada, abrangendo as cinco regiões geográficas. A PPV também coletou dados de morbidade e utilização de serviços de saúde, 
porém restringiu-se às regiões Nordeste e Sudeste. Neste trabalho, analisaram-se os dados das regiões Nordeste e Sudeste das duas pesquisas, tendo sido excluídos de ambas as amostras os domicílios situados em área rural. Maiores detalhes sobre as características do plano amostral e das variáveis de cada pesquisa encontram-se em Fletcher (1988) e IBGE (1998).

Com o objetivo de investigar a ocorrência de desigualdades geográficas no consumo de serviços de saúde, calcularam-se taxas de utilização de serviços, padronizadas por idade e sexo, para as pessoas com e sem morbidade e para as pessoas com e sem plano de saúde. $\mathrm{O}$ processo de padronização foi baseado no método direto, e a população do Sudeste foi utilizada como padrão. Comparou-se também a dimensão do gasto privado domiciliar com medicamentos e com planos de saúde, entre as regiões e os grupos de renda. Para avaliar a existência de desigualdades sociais no uso de serviços de saúde estimou-se a razão de odds para três grupos de renda e para as pessoas com e sem cobertura de plano de saúde. O procedimento estatístico utilizado foi a regressão logística e o modelo de predição de uso de serviços de saúde incluiu as variáveis idade, sexo, morbidade (restrição de atividades) e tercil de renda domiciliar per capita ou cobertura de plano de saúde. Por fim, estudaram-se as variações na estrutura de consumo de serviços de saúde, nas dimensões de eqüidade geográfica e social, com base na distribuição proporcional do uso de serviços de saúde segundo o tipo de estabelecimento utilizado.

A fim de analisar a ocorrência ou não de mudança no padrão de eqüidade no consumo de serviços de saúde, após a implantação do SUS, procedeu-se à comparação entre os resultados de cada pesquisa. É preciso destacar, no entanto, que estas comparações apresentam limitações devidas a diferenças no desenho amostral e na definição das variáveis em cada um dos estudos. Desta forma, as análises centram-se principalmente na observação de mudança no padrão de eqüidade geográfica e/ou social em cada período, evitando-se a comparação direta da magnitude dos parâmetros entre as pesquisas.

As variáveis consideradas como proxy de morbidade estão definidas no quadro 1 .

A variável social escolhida foi a renda domiciliar per capita. As duas pesquisas empregaram metodologias diversas para gerar a infor- mação sobre renda. Como na PNSN utiliza-se apenas a renda monetária auferida nos últimos trinta dias - e proveniente de trabalho, aposentadoria, abono, pensão alimentícia, aluguel, doação, juros e dividendos -, procurouse, no caso da PPV, cujas informações são mais abrangentes, trabalhar com itens análogos ao da PNSN. Em cada pesquisa, os domicílios foram classificados em tercis de renda definidos segundo a distribuição da renda domiciliar per capita para o conjunto das duas regiões.

A variável cobertura de plano de saúde, disponível apenas na PPV, discrimina as pessoas segundo a participação em convênios ou seguros de saúde privados.

O uso de serviços de saúde, variável dicotômica, indica se o indivíduo foi ou não atendido em serviço de saúde em um período determinado. Na PNSN, corresponde aos quinze dias que antecederam a entrevista; na PPV, corresponde a trinta dias. A estrutura do consumo foi analisada através do uso segundo o tipo de estabelecimento. Com o objetivo de possibilitar a comparação entre as pesquisas, os tipos de estabelecimentos foram recodificados nas categorias apresentadas no quadro 2.

Ainda assim, não foi possível criar categorias homogêneas no caso do atendimento ambulatorial, já que a PPV incorporou à categoria Posto ou Centro de Saúde os atendimentos realizados nos Postos de Assistência Médica (PAM), que na PNSN pertencem à categoria Clínica ou posto de assistência.

Finalmente, para analisar o gasto em saúde, informação disponível apenas na PPV, trabalhou-se somente com os gastos com remédios e seguro-saúde, para os quais considerouse adequado o período de referência adotado pela pesquisa - trinta dias anteriores à entrevista. O mesmo não se pode dizer para gastos com consultas, internação, exames laboratoriais e radiológicos que, por serem itens de consumo menos freqüentes, necessitariam de um período maior de referência.

O tratamento estatístico dos dados resultantes de inquéritos de grande porte, tais como a PNSN e a PPV, requer cuidados especiais. A hipótese de amostra aleatória simples com reposição (AASC), utilizada nos procedimentos estatísticos dos programas convencionais (SPSS, SAS, BMDP etc.), é claramente inadequada para o tratamento dos dados de pesquisas por amostragem complexa, como é o caso das amostras da PNSN e da PPV (Pessoa \& Silva, 1998). O impacto do desenho amostral na 


\section{Quadro 1}

Variáveis de morbidade, Pesquisa Nacional sobre Saúde e Nutrição e Pesquisa sobre Padrões de Vida (PPV).

\section{PNSN}

Restrição de atividades rotineiras por motivo de doença nos quinze dias que antecederam a entrevista.

Sintoma: variável dicotômica criada a partir das respostas sobre ocorrência ou não de uma lista de doenças e sintomas nos quinze dias que antecederam a entrevista.

\section{PPV}

Restrição de atividades rotineiras por motivo de doença nos trinta dias que antecederam a entrevista.

Doença crônica: variável dicotômica criada a partir das respostas sobre ocorrência ou não de uma lista de doenças crônicas selecionadas.

Presença de problema de saúde: ocorrência ou não de algum problema de saúde nos trinta dias que antecederam a entrevista.

Auto-avaliação do estado de saúde: variável criada recodificando-se as categorias excelente, muito bom e bom, como bom estado de saúde e as categorias regular e ruim, como estado de saúde deficiente.

\section{Quadro 2}

Categorias e serviços de saúde, Pesquisa Nacional sobre Saúde e Nutrição e Pesquisa sobre Padrões de Vida (PPV).

\begin{tabular}{lll}
\hline Categoria agregada & PNSN & PPV \\
\hline Hospital & Hospital & Hospital público \\
& Pronto-socorro & Hospital particular \\
& Hospital particular conveniado \\
Posto ou & Posto ou & Posto ou \\
Centro de Saúde & Centro de Saúde & Centro de Saúde \\
Clínica ou & Clínica ou & Clínica particular \\
consultório particular & posto de assistência & conveniada \\
& Consultório ou & Clínica ou \\
Farmácia e outros & médico particular & consultório particular \\
& Farmácia & Farmácia \\
& Outros & No próprio domicílio \\
\hline
\end{tabular}

variância dos estimadores da regressão logística pode ser capturado pelo efeito de delineamento, que é a razão entre a variância calculada assumindo a amostra complexa e a variância assumindo a amostra aleatória simples. Portanto, quando o efeito de delineamento para um determinado estimador é diferente de 1 , pode-se concluir que houve impacto do plano amostral no teste de significância do coeficiente associado à variável em questão, indicando a necessidade de se calcular a variância assumindo a amostra complexa.
Com o objetivo de incorporar o efeito de delineamento da amostra na modelagem estatística dos dados da PNSN e da PPV, utilizou-se o SUDAAN (Shah et al., 1997), um dos programas estatísticos comerciais disponíveis para o tratamento de dados oriundos de pesquisa com amostragem complexa. Entre os esquemas amostrais disponíveis no SUDAAN, utilizou-se aquele mais adequado às características amostrais da PNSN e PPV - o desenho WR (com reposição). Esse desenho é apropriado para amostragem com reposição no pri- 
meiro estágio e amostragem com ou sem reposição nos estágios subseqüentes, sendo as probabilidades de reposição iguais ou diferentes em qualquer um dos estágios.

Para contornar o problema de erro nas estimativas pontuais calculadas para médias, proporções, coeficientes de regressão, entre outros, devido a probabilidades distintas de seleção dos elementos da amostra e da ocorrência de não respostas, utilizou-se, nos procedimentos estatísticos realizados, os pesos amostrais disponíveis nos bancos de dados originais. Estes pesos foram corrigidos para evitar a expansão da amostra, pois os grandes números resultantes do uso dos pesos não corrigidos implicaria aumento da ocorrência de significância estatística. Com essa correção, a composição manteve-se nas análises o número de pessoas e de domicílios efetivamente pesquisadas em cada uma das regiões.

\section{Resultados}

As regiões geográficas analisadas encontramse em posições polares, em relação às demais: o Sudeste - a região mais desenvolvida - e o Nordeste - a menos desenvolvida. Em 1989, o Sudeste, comparativamente ao Nordeste, apresentava uma maior razão de sexos (total de homens/total de mulheres) - 0,96 e 0,88 respectivamente -, um perfil etário mais envelhecido, melhor nível de escolaridade. Observa-se ainda que a população do Sudeste era predominantemente de raça branca $(65,62 \%)$, ao passo que a do Nordeste era predominantemente de raça não branca $(78,58 \%)$. O coeficiente de Gini indicava menor concentração de renda no Sudeste, apesar de ambas as regiões apresentarem marcadas desigualdades (Quadro 3).

Em 1996/1997, entretanto, observa-se uma convergência nas razões de sexos entre o $\mathrm{Su}$ deste e o Nordeste (0,93 e 0,91 respectivamente), e o perfil etário de cada região assume características compatíveis com estruturas populacionais menos jovens, em comparação com o perfil etário observado em 1989. A proporção de chefes de família sem instrução ou com instrução elementar $(34,65 \%$ no Sudeste e $35,9 \%$ no Nordeste) reduziu-se em comparação com o ano de 1989, observando-se, também, redução na razão da proporção de chefes de família sem instrução ou com nível elementar de instrução entre as duas regiões $(1,2$ em 1989 e 1,04 em 1996/1997). A concentração de renda apresenta uma diminuição no Nordeste e um ligeiro aumento no Sudeste, implicando diminuição das diferenças na concentração de renda.

Os altos valores do efeito de delineamento (ED), observados em algumas variáveis, refletem a alta correlação entre indivíduos em cada estrato amostral, confirmando a necessidade de se utilizar procedimentos que corrijam o impacto do desenho amostral no teste de significância estatística como os disponíveis no SUDAAN (Quadro 3).

Com relação ao perfil de necessidades de saúde, em 1989, observa-se variação estatisticamente significativa na proporção de pessoas que mencionaram restrição de atividade por motivo de saúde, nas duas regiões, desfavoráveis ao Sudeste. Porém, com a variável sintoma não se verifica diferença estatisticamente significativa. O mesmo padrão não se reproduz em 1996/1997, quando as desigualdades se invertem, passando a população do Nordeste a apresentar valores significativamente maiores em todos os indicadores de morbidade estudados, com exceção da proporção de pessoas que referiram doenças crônicas, o que não apresenta diferença estatisticamente significativa entre as duas regiões (Quadro 4).

Observa-se ainda que, em 1989, as chances de um indivíduo mencionar restrição de atividades rotineiras por motivo de saúde, tanto no Nordeste quanto no Sudeste, eram maiores no tercil mais baixo de renda domiciliar per capita, comparativamente ao tercil mais alto. Tomando-se como referência para morbidade a variável auto-avaliação das condições de saúde, observa-se, em 1996/1997, um gradiente social bastante evidente, também desfavorável aos grupos de menor renda. A razão de odds entre o primeiro e o terceiro tercil foi 3,07 no Nordeste e 2,06 no Sudeste (Quadro 5).

Ao se analisarem as variações geográficas nas taxas de utilização de serviços de saúde padronizadas por idade e sexo, observa-se uma posição desfavorável para o Nordeste $(13,01$ por 100 habitantes), em 1989, com o Sudeste apresentando uma maior taxa de utilização de serviços de saúde de 19,49 por 100 habitantes. Em 1996/1997 as diferenças nas regiões deixam de ser estatisticamente significativas (Quadro 4).

Porém, quando se recalculam as taxas de utilização mencionadas para os grupos com e 


\section{Quadro 3}

Características demográficas e socioeconômicas. Nordeste e Sudeste - 1989 e 1996/1997.

\begin{tabular}{|c|c|c|c|c|c|c|c|c|c|c|c|c|}
\hline & \multicolumn{5}{|c|}{1989} & \multicolumn{7}{|c|}{$1996 / 1997$} \\
\hline & \multicolumn{3}{|c|}{ Nordeste } & \multicolumn{3}{|c|}{ Sudeste } & \multicolumn{3}{|c|}{ Nordeste } & \multicolumn{3}{|c|}{ Sudeste } \\
\hline & $\%$ & $\begin{array}{l}\text { Erro } \\
\text { padrão }\end{array}$ & $\mathrm{ED}^{\star}$ & $\%$ & $\begin{array}{l}\text { Erro } \\
\text { padrão }\end{array}$ & ED & $\%$ & $\begin{array}{l}\text { Erro } \\
\text { padrão }\end{array}$ & ED & $\%$ & $\begin{array}{l}\text { Erro } \\
\text { padrão }\end{array}$ & ED \\
\hline \multicolumn{13}{|l|}{ Sexo } \\
\hline Mulheres & 53,06 & $(0,68)$ & 1,35 & 50,87 & $(0,51)$ & 0,66 & 52,30 & $(0,75)$ & 1,57 & 51,67 & $(0,60)$ & 1,18 \\
\hline Homens & 46,94 & $(0,68)$ & 1,35 & 49,13 & $(0,51)$ & 0,66 & 47,70 & $(0,75)$ & 1,57 & 48,33 & $(0,60)$ & 1,18 \\
\hline \multicolumn{13}{|l|}{ Raça } \\
\hline Branca & 21,42 & $(2,68)$ & 31,36 & 65,62 & $(3,20)$ & 28,78 & 29,92 & $(2,22)$ & 16,15 & 71,81 & $(2,27)$ & 20,81 \\
\hline Não branca & 78,58 & $(2,68)$ & 31,36 & 34,38 & $(3,20)$ & 28,78 & 70,08 & $(2,22)$ & 16,15 & 28,19 & $(2,27)$ & 20,81 \\
\hline \multicolumn{13}{|l|}{ Idade } \\
\hline 0 a 4 & 12,44 & $(0,60)$ & 2,42 & 10,00 & $(0,52)$ & 1,91 & 11,62 & $(0,55)$ & 2,00 & 8,93 & $(0,48)$ & 2,29 \\
\hline 5 a 19 & 36,66 & $(0,86)$ & 2,35 & 31,54 & $(1,33)$ & 5,21 & 34,21 & $(0,83)$ & 2,11 & 29,32 & $(0,71)$ & 1,97 \\
\hline 20 a 59 & 41,94 & $(0,92)$ & 2,55 & 50,90 & $(0,90)$ & 2,40 & 46,02 & $(0,79)$ & 1,74 & 52,21 & $(0,62)$ & 1,28 \\
\hline $60 e+$ & 8,96 & $(0,62)$ & 3,44 & 7,55 & $(0,72)$ & 4,64 & 8,15 & $(0,61)$ & 3,45 & 9,54 & $(0,61)$ & 3,49 \\
\hline \multicolumn{13}{|c|}{ Escolaridade do chefe } \\
\hline $\begin{array}{l}\text { Nenhuma ou } \\
\text { elementar }\end{array}$ & 66,03 & $(2,76)$ & 25,01 & 55,06 & $(2,78)$ & 19,72 & 35,99 & $(1,92)$ & 10,95 & 34,65 & $(1,41)$ & 7,16 \\
\hline $\begin{array}{l}\text { Primeiro grau } \\
\text { completo }\end{array}$ & 19,04 & $(1,70)$ & 13,71 & 24,85 & $(1,63)$ & 9,04 & 39,03 & $(1,80)$ & 9,33 & 36,24 & $(1,51)$ & 8,10 \\
\hline $\begin{array}{l}\text { Segundo grau } \\
\text { ou superior }\end{array}$ & 14,92 & $(2,21)$ & 28,30 & 20,09 & $(2,58)$ & 26,26 & 24,98 & $(1,63)$ & 9,73 & 29,11 & $(1,51)$ & 9,07 \\
\hline
\end{tabular}

Cobertura de plano de saúde

$17,09 \quad(1,36) \quad 8,91 \quad 34,44 \quad(1,65) \quad 9,85$

\section{Coeficiente de Gini}

$\begin{array}{llll}0,6687 & 0,5616 & 0,6029 & 0,5885\end{array}$

* Efeito de delineamento da amostra.

Fonte: IBGE - Pesquisa Nacional sobre Saúde e Nutrição (1989) e Pesquisa sobre Padrões de Vida (1996/1997).

sem morbidade (restrição de atividades), observam-se marcadas diferenças entre as regiões, estatisticamente significativas e sempre desfavoráveis para o Nordeste, no ano de 1989, em particular (Quadro 6). As taxas de utilização dos indivíduos com morbidade são sistematicamente maiores do que as taxas dos indivíduos sem morbidade, confirmando a existência de associação positiva entre uso de serviços de saúde e presença de problemas de saúde. Por outro lado, os dados sugerem uma redução das desigualdades geográficas no uso de serviços no período de estudo. Entre 1989 e 1996/1997, a razão das taxas de utilização entre o Sudeste e o Nordeste diminuiu de 1,42, em 1989, para 1,17, em 1996/1997, para os indivíduos que referiram morbidade (restrição de atividade), e de 1,42 para 1,25, respectivamente, para os que não referiram morbidade.

A comparação das taxas de utilização nos dois períodos de estudo, para as pessoas classificadas nas demais variáveis proxy de morbidade, não pôde ser efetuada, pois estas variáveis não estão disponíveis em ambas as pesquisas. No entanto, em cada uma delas, essas taxas também expressam um padrão de desigualdade geográfica no uso de serviços, desfavorável ao Nordeste (Quadro 6). 
Quadro 4

Taxas de morbidade (por 100 habitantes) e taxas de utilização de serviços de saúde, padronizadas por sexo e idade. Nordeste e Sudeste - 1989 e 1996/1997.

\begin{tabular}{|c|c|c|c|c|c|c|c|c|}
\hline & \multicolumn{4}{|c|}{1989} & \multicolumn{4}{|c|}{ 1996/1997 } \\
\hline & \multicolumn{2}{|c|}{ Nordeste } & \multicolumn{2}{|c|}{ Sudeste } & \multicolumn{2}{|c|}{ Nordeste } & \multicolumn{2}{|c|}{ Sudeste } \\
\hline & $\%$ & $\begin{array}{l}\text { Erro } \\
\text { padrão }\end{array}$ & $\%$ & $\begin{array}{l}\text { Erro } \\
\text { padrão }\end{array}$ & $\%$ & $\begin{array}{l}\text { Erro } \\
\text { padrão }\end{array}$ & $\%$ & $\begin{array}{l}\text { Erro } \\
\text { padrão }\end{array}$ \\
\hline $\begin{array}{l}\text { Com restrição } \\
\text { de atividade }\end{array}$ & 7,80 & $(0,61)$ & 9,78 & $(0,57)^{\star}$ & 9,74 & $(0,87)$ & 7,16 & $(0,59)^{\star}$ \\
\hline $\begin{array}{l}\text { Com doença } \\
\text { crônica }\end{array}$ & - & & - & & 15,00 & $(0,93)$ & 14,91 & $(0,67)^{\mathrm{ns}}$ \\
\hline $\begin{array}{l}\text { Com problema } \\
\text { de saúde }\end{array}$ & - & & - & & 26,01 & $(1,99)$ & 18,93 & $(1,02)^{\star \star *}$ \\
\hline $\begin{array}{l}\text { Auto-avaliação } \\
\text { regular ou ruim }\end{array}$ & - & & - & & 22,32 & $(1,25)$ & 15,37 & $(0,84)^{\star * *}$ \\
\hline Com sintoma & 53,58 & $(2,36)$ & 51,48 & $(1,94) \mathrm{ns}$ & - & & - & \\
\hline $\begin{array}{l}\text { Uso de serviços } \\
\text { de saúde }\end{array}$ & 13,01 & $(0,90)$ & 19,49 & $(0,81)^{\star}$ & 14,06 & $(0,88)$ & 15,29 & $(0,97) \mathrm{ns}$ \\
\hline
\end{tabular}

ns: não significativo.

${ }^{\star} 0,01<\mathrm{p}$-valor $<0,05$.

$* *$ p-valor $<0,01$.

Fonte: IBGE - Pesquisa Nacional sobre Saúde e Nutrição (1989) e Pesquisa sobre Padrões de Vida (1996/1997).

Quadro 5

Razão de odds para morbidade condicional à renda domiciliar per capita.

\begin{tabular}{|c|c|c|c|c|c|c|c|}
\hline \multirow[b]{2}{*}{ Característica } & \multirow[b]{2}{*}{ Região } & \multicolumn{3}{|c|}{ PNSN $1989^{* *}$} & \multicolumn{3}{|c|}{ PPV 1996/97*** } \\
\hline & & $\begin{array}{l}\text { Razão } \\
\text { de odds }\end{array}$ & $\begin{array}{l}\text { Limite } \\
\text { inferior }\end{array}$ & $\begin{array}{l}\text { Limite } \\
\text { superior }\end{array}$ & $\begin{array}{l}\text { Razão } \\
\text { de odds }\end{array}$ & $\begin{array}{l}\text { Limite } \\
\text { inferior }\end{array}$ & $\begin{array}{l}\text { Limite } \\
\text { superior }\end{array}$ \\
\hline \multicolumn{8}{|c|}{$\begin{array}{l}\text { Renda domiciliar } \\
\text { per capita }\end{array}$} \\
\hline \multicolumn{8}{|l|}{ Primeiro tercil } \\
\hline & $\mathrm{NE}$ & 1.24 & 0.99 & 1.57 & 3.07 & 2.22 & 4.25 \\
\hline & SE & 1.15 & 0.94 & 1.40 & 2.06 & 1.51 & 2.80 \\
\hline \multicolumn{8}{|l|}{ Segundo tercil } \\
\hline & $\mathrm{NE}$ & 1.39 & 0.99 & 1.97 & 2.53 & 1.79 & 3.59 \\
\hline & SE & 1.18 & 0.94 & 1.48 & 1.41 & 1.12 & 1.79 \\
\hline
\end{tabular}

* Intervalo de confiança 95\%.

** Restrição de atividade.

*** Auto-avaliação do estado de saúde.

As variações sociais na utilização de serviços de saúde demonstram que condições sociais desfavoráveis tendem a reduzir as chances de uso de serviços. O efeito do aumento da renda no consumo de serviços de saúde, controlado por idade, sexo e morbidade (restrição de atividades) está presente em ambas as regiões e em ambas as pesquisas. Por exemplo, no Nordeste, as chances dos indivíduos pertencentes ao primeiro tercil de renda, em relação aos indivíduos do terceiro tercil, é 52\% menor em 1989 (razão de odds =0,48) e 37\% menor em 1996/1997 (razão de odds $=0,63$ ). Da mesma forma, no Sudeste, as chances de 
indivíduos do primeiro tercil utilizarem serviços de saúde são $40 \%$ e $35 \%$ menores do que a dos indivíduos do terceiro tercil (razões de odds $=0,60$ e 0,65), respectivamente, em 1989 e 1996/1997 (Quadro 7).

A cobertura por plano de saúde, informação disponível apenas para 1996/1997, constitui uma outra dimensão para a análise da eqüidade no uso de serviços de saúde. Esta cobertura apresenta variação importante entre as regiões, sendo duas vezes maior no Sudeste $(34,44 \%)$ do que no Nordeste $(17,09 \%)$ (Quadro 3$)$, e cresce marcadamente com a renda, em ambas as regiões, caracterizando um padrão de expressivas desigualdades geográfica e social. A razão da proporção de população coberta por plano de saúde entre o terceiro e o primeiro tercil de renda é igual a 16,5 no Nordeste e 11,3 no Sudeste.

A taxa de utilização padronizada por sexo e idade segundo a cobertura de plano de saúde não apresenta variação estatisticamente significativa entre as duas regiões, mas é maior no grupo de pessoas cobertas por planos de saúde (18,93 por 100 habitantes no Nordeste e 18,20 por 100 habitantes no Sudeste) do que no grupo de pessoas não cobertas (13,19 por 100 habitantes no Nordeste e 13,72 por 100 habitantes no Sudeste). A chance de uso de serviços de saúde, controlada por idade, sexo e morbidade (restrição de atividades), é expressivamente maior para as pessoas cobertas por planos de saúde em relação às pessoas não cobertas $-66 \%$ no Nordeste e $73 \%$ no Sudeste (razão de $o d d s=1,66$ e 1,73 respectivamente) (Quadro 7).

Em relação à estrutura de consumo de serviços de saúde em 1989 percebe-se importantes variações regionais: maior participação relativa de postos e centros de saúde no Nordeste $(29,01 \%$ - Nordeste, $16,75 \%$ - Sudeste $)$ contra maior participação relativa de clínicas e consultórios particulares no sudeste $(29,34 \%$ Nordeste, 45,10\% - Sudeste). Varia também a estrutura de consumo antes e após a implantação do SUS. O que chama atenção nesta va-

\section{Quadro 6}

Taxas de utilização de serviços de saúde (por 100 habitantes), padronizadas por idade e sexo, por indicadores de morbidade e cobertura de plano de saúde, segundo a região Nordeste e Sudeste, 1989 e 1996/1997.

\begin{tabular}{|c|c|c|c|c|c|c|c|}
\hline & \multicolumn{3}{|c|}{1989} & \multicolumn{4}{|c|}{ 1996/1997 } \\
\hline & \multicolumn{2}{|c|}{ Nordeste } & Sudeste & \multicolumn{2}{|c|}{ Nordeste } & \multicolumn{2}{|c|}{ Sudeste } \\
\hline & $\%$ & $\begin{array}{l}\text { Erro } \\
\text { padrão }\end{array}$ & $\begin{array}{ll}\% & \text { Erro } \\
& \text { padrão }\end{array}$ & $\%$ & $\begin{array}{l}\text { Erro } \\
\text { padrão }\end{array}$ & $\%$ & $\begin{array}{l}\text { Erro } \\
\text { padrão }\end{array}$ \\
\hline \multicolumn{8}{|c|}{ Restrição de atividade } \\
\hline com restrição & 46,39 & $(3,10)$ & $66,02(2,62)^{* *}$ & 59,57 & $(2,80)$ & 69,54 & $(3,53)^{*}$ \\
\hline sem restrição & 10,23 & $(0,88)$ & $14,55(0,79)^{* *}$ & 9,04 & $(0,69)$ & 11,32 & $(0,90)^{*}$ \\
\hline \multicolumn{8}{|c|}{ Problema de saúde } \\
\hline com problema & - & - & $36,82(2,51)$ & 52,14 & $(2,11)^{\star \star}$ & & \\
\hline sem problema & - & - & $4,96 \quad(0,55)$ & 6,00 & $(0,69) \mathrm{ns}$ & & \\
\hline
\end{tabular}

\begin{tabular}{lllllll}
\hline Problema de saúde & & & & & \\
\hline com sintoma & 20,53 & $(1,61)$ & 30,67 & $(1,17)^{\star *}$ & - & - \\
sem sintoma & 3,19 & $(0,53)$ & 6,64 & $(0,63)^{\star *}$ & - & - \\
\hline
\end{tabular}

\section{Plano de saúde}

\begin{tabular}{lllll}
\hline coberto & - & - & $18,93(1,73)$ & $18,20(1,52) \mathrm{ns}$ \\
não coberto & - & - & $13,19(0,95)$ & $13,72 \quad(1,04) \mathrm{ns}$ \\
\hline
\end{tabular}

ns: não significativo.

$\star 0,01<\mathrm{p}$-valor $<0,05$.

** p-valor $<0,01$

Fonte: IBGE - Pesquisa Nacional sobre Saúde e Nutrição (1989) e Pesquisa sobre Padrões de Vida (1996/1997). 
Quadro 7

Razão de odds para uso de serviços de saúde condicional à renda domiciliar per capita e cobertura por plano de saúde.

\begin{tabular}{|c|c|c|c|c|c|c|c|}
\hline \multirow[b]{2}{*}{ Característica } & \multirow[b]{2}{*}{ Região } & \multicolumn{3}{|c|}{ PNSN 1989** } & \multicolumn{3}{|c|}{ PPV $1996 / 97^{* * *}$} \\
\hline & & $\begin{array}{l}\text { Razão } \\
\text { de odds }\end{array}$ & $\begin{array}{l}\text { Limite } \\
\text { inferior }\end{array}$ & $\begin{array}{l}\text { Limite } \\
\text { superior }\end{array}$ & $\begin{array}{l}\text { Razão } \\
\text { de odds }\end{array}$ & $\begin{array}{l}\text { Limite } \\
\text { inferior }\end{array}$ & $\begin{array}{l}\text { Limite } \\
\text { superior }\end{array}$ \\
\hline \multicolumn{8}{|c|}{$\begin{array}{l}\text { Renda domiciliar } \\
\text { per capita }\end{array}$} \\
\hline \multicolumn{8}{|l|}{ Primeiro tercil } \\
\hline & $\mathrm{NE}$ & 0.48 & 0.34 & 0.66 & 0.63 & 0.46 & 0.85 \\
\hline & SE & 0.60 & 0.46 & 0.79 & 0.65 & 0.48 & 0.88 \\
\hline \multicolumn{8}{|l|}{ Segundo tercil } \\
\hline & $\mathrm{NE}$ & 0.73 & 0.52 & 1.01 & 0.78 & 0.49 & 1.23 \\
\hline & SE & 0.91 & 0.74 & 1.12 & 0.61 & 0.48 & 0.79 \\
\hline \multicolumn{8}{|l|}{$\begin{array}{l}\text { Cobertura por } \\
\text { plano de saúde }\end{array}$} \\
\hline \multicolumn{8}{|l|}{ Coberto } \\
\hline & $\mathrm{NE}$ & - & - & - & 1.66 & 1.26 & 2.20 \\
\hline & SE & - & - & - & 1.73 & 1.38 & 2.17 \\
\hline
\end{tabular}

* Intervalo de confiança 95\%.

riação é o aumento expressivo da participação relativa do hospital no consumo de serviços de saúde, em ambas as regiões, tendência esta mais evidente no Nordeste. O aumento da participação dos hospitais, em particular no Nordeste, fez-se em detrimento da participação dos postos e centros de saúde, e da farmácia e outros (Figuras 1 e 2).

Ao se analisar a estrutura de consumo de serviços entre os grupos de renda, observa-se também uma marcada variação em ambas as regiões: os grupos de mais alta renda consomem mais serviços ambulatoriais e o consumo de serviços hospitalares tende a aumentar à medida em que a renda decresce. Além disso, há uma variação no tipo de serviço ambulatorial utilizado: os grupos de maior renda usam predominantemente consultórios particulares e clínicas, ao passo que os de menor renda usam mais postos e centros de saúde. Vale destacar que a participação relativa no consumo de serviços hospitalares é observada em todos os grupos de renda, sendo mais acentuada no tercil de mais baixa renda do Nordeste e após a implantação do SUS, quando o uso de serviços hospitalares representou pouco mais de $62 \%$ do consumo desse grupo (Figura 3).
A estrutura de consumo de serviços de saúde entre as pessoas cobertas e não cobertas por plano de saúde também varia. O uso de clínicas e consultórios particulares predomina no grupo de indivíduos com plano de saúde, ao passo que o uso de hospitais e postos/centros de saúde é maior no grupo de indivíduos não cobertos. Este padrão é semelhante nas duas regiões analisadas (Figura 4).

O gasto médio com planos de saúde entre os domicílios que referiram esse tipo de despesa é semelhante no Nordeste $(\mathrm{R} \$ 321,03)$ e no Sudeste $(\mathrm{R} \$ 312,80)$. Ainda que a análise se ressinta do pequeno número de observações, particularmente no primeiro tercil de renda no Sudeste, as magnitudes das diferenças permitem inferir que o gasto com planos de saúde tende a aumentar com a renda nas duas regiões. Entretanto, verifica-se que a importância desse tipo de despesa no orçamento domiciliar é tanto maior quanto menor a renda. As ressalvas metodológicas quanto ao número de observações também devem ser notadas nesse caso, em particular no primeiro tercil do $\mathrm{Su}$ deste (Quadro 8).

Analogamente, o gasto médio com medicamentos por domicílio foi de $\mathrm{R} \$ 23,73$ no Sudeste e de R\$15,36 no Nordeste. A diferença 
entre as regiões é estatisticamente significante $(p$-valor $=0.0204)$. Por outro lado, os gastos médios variam positivamente com a renda, sendo as diferenças entre as médias do primeiro e do terceiro tercil de renda estatisticamente significantes no Nordeste $(\mathrm{p}$-valor $=0,0012)$ e no Sudeste $(p$-valor $=0,0036)$. Assim como no caso do gasto com planos de saúde, a proporção do gasto com medicamentos na renda domiciliar varia inversamente ao aumento da renda. Os residentes nos domicílios de menor renda gastam menos com medicamentos, mas a participação relativa deste gasto na renda domiciliar é expressivamente maior (Quadro 8).

\section{Discussão}

Os resultados aqui apresentados evidenciam a multiplicidade de fatores que interferem no padrão de consumo de serviços de saúde e o imbricamento perverso entre eles no Brasil, resultando em um quadro de desigualdades cumulativas que evidenciam o quão distante encontra-se o sistema de saúde do país dos princípios igualitários enunciados na sua formulação.

Com relação às desigualdades no adoecer (necessidade em saúde) o Nordeste - região menos desenvolvida - apresenta, em 1996/ 1997, taxas mais altas de morbidade compa- rativamente ao Sudeste. É importante ressaltar que, nos dados de 1989, o perfil de morbidade observado entre as regiões era o inverso, isto é, desfavorável para o Sudeste. Porém, os dados de 1996/1997 são bastante consistentes, já que o mesmo comportamento -taxas mais elevadas no Nordeste - é observado nas diversas variáveis de morbidade, padrão este consistente com a literatura que aponta associação inversa entre condições de vida e saúde (Black et al., 1990; Marmot, 1986). Uma possível limitação na qualidade das respostas dos entrevistados do Nordeste à variável 'restrição de atividades', na pesquisa de 1989, pode explicar os resultados obtidos, não sendo recomendável assumir que houve inversão no padrão de morbidade entre o Nordeste e o Sudeste no período de análise. Além disso, os dados da PNSN e da PPV também indicam que os indivíduos mais pobres têm maior chance de adoecer, padrão verdadeiro para ambos os períodos nas duas regiões.

A comparação das taxas de utilização padronizadas por idade e sexo entre regiões e/ou grupos sociais, com objetivo de verificar a existência de desigualdades, deve ser controlada pela presença ou não de morbidade, já que ela é um importante preditor de uso de serviços de saúde e sua ocorrência varia entre as regiões e os grupos sociais (O'Donnell \& Propper, 1989). Isto pode ser verificado neste estudo,

\section{Quadro 8}

Gasto médio domiciliar com plano de saúde e remédios segundo tercil de renda domiciliar per capita.

\begin{tabular}{|c|c|c|c|c|c|c|c|c|}
\hline \multirow[b]{2}{*}{$\begin{array}{l}\text { Tercil de renda } \\
\text { domiciliar } \\
\text { per capita }\end{array}$} & \multicolumn{3}{|c|}{ Gasto médio com Plano de Saúde } & \multirow[b]{2}{*}{$(\mathbf{a} / \mathbf{b} \%)$} & \multicolumn{4}{|c|}{ Gasto médio com medicamentos } \\
\hline & $\begin{array}{l}\text { Domicílios } \\
\text { que referiram } \\
\text { gasto }\end{array}$ & $\begin{array}{l}\text { Gasto } \\
\text { médio } \\
(\mathrm{R} \$)(\mathrm{a})\end{array}$ & $\begin{array}{l}\text { Renda média } \\
\text { domiciliar } \\
(\mathrm{R} \$)(\mathrm{b})\end{array}$ & & Domicílios & $\begin{array}{l}\text { Gasto } \\
\text { médio } \\
(\mathrm{R} \$)(\mathrm{c})\end{array}$ & $\begin{array}{l}\text { Renda média } \\
\text { domiciliar } \\
(\mathrm{R} \$)(\mathrm{d})\end{array}$ & $(\mathrm{c} / \mathrm{d} \%)$ \\
\hline \multicolumn{9}{|l|}{ Nordeste } \\
\hline 1 & 25 & 98,7 & 267,2 & 36.9 & 917 & 9,22 & 279,64 & 3,30 \\
\hline 2 & 40 & 181,5 & 920,0 & 19.7 & 448 & 14,67 & 689,00 & 2,13 \\
\hline 3 & 221 & 371,2 & 4656,7 & 8.0 & 589 & 25,70 & 3163,76 & 0,81 \\
\hline Total & 286 & 321,0 & 3753,7 & 8.5 & 1954 & 15,36 & 1233,59 & 1,25 \\
\hline \multicolumn{9}{|l|}{ Sudeste } \\
\hline 1 & 14 & 201.6 & 238,4 & 84.6 & 407 & 13,23 & 312,18 & 4,24 \\
\hline 2 & 56 & 123.0 & 775,8 & 15.9 & 488 & 17,20 & 723,61 & 2,38 \\
\hline 3 & 251 & 361.4 & 4408,9 & 8.2 & 1064 & 31,45 & 2889,24 & 1,09 \\
\hline Total & 321 & 312.8 & 3594,5 & 8.7 & 1959 & 23,73 & 1758,59 & 1,35 \\
\hline
\end{tabular}

Fonte: IBGE-PPV, 1996/97. 
Figura 1

Taxa proporcional de uso de serviços de saúde, padronizada por idade e sexo, por região, segundo tipo de estabelecimento, 1989.

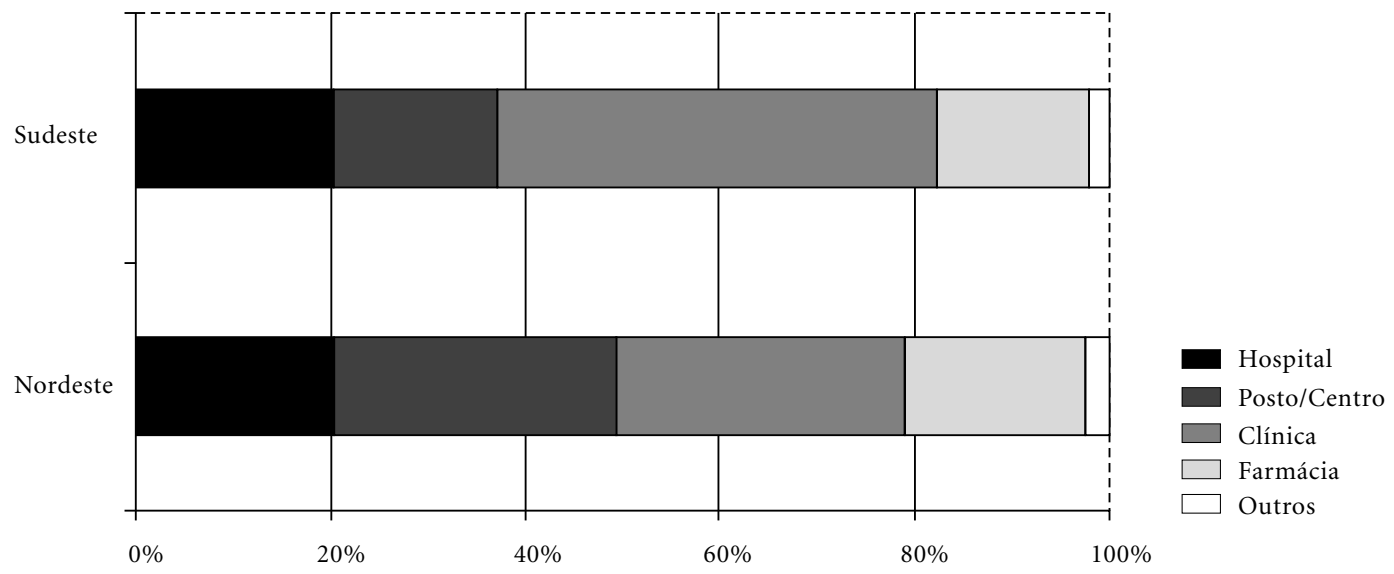

já que as diferenças entre as regiões ficam evidentes após a incorporação na análise da variável morbidade. As desigualdades geográficas no consumo de serviços de saúde, desfavoráveis ao Nordeste em 1989, tanto para as pessoas que referiram morbidade como para aquelas que não referiram, mantêm-se após a implantação do SUS. Porém, os valores das taxas sugerem uma redução nas diferenças regionais entre os períodos estudados. Almeida et al. (1999b) já tinham mostrado que, com relação às taxas de internação hospitalar, ocorreu, entre 1993 e 1996, alguma redução nas desigualdades geográficas em favor do Nordeste.

Neste estudo também se demonstra que, ceteris paribus, as pessoas mais pobres têm menor chance de consumir serviços de saúde comparativamente às mais ricas e que este padrão permanece entre os dois períodos analisados. Além disso, constata-se que as chances de adoecer diminuem com o aumento da renda, o inverso ocorrendo com o consumo de serviços de saúde, fato verdadeiro para ambas as regiões e nos dois períodos de análise, indicando que o sistema de saúde brasileiro permanece apresentando marcadas desigualdades sociais, caracterizadas pelo fato de que as pessoas que mais necessitam têm menor chance de receber atendimento. Mesmo assim, a diminuição dos valores das razões de odds entre os grupos de renda no ano de 1996/1997 sugere que a política de saúde recente produziu algum impacto na direção de uma maior equidade.

A participação do seguro privado no sistema de saúde brasileiro também atua no sentido de acentuar as desigualdades no consumo de serviços de saúde descritas acima. A cobertura de plano de saúde é dependente da renda, sendo expressivamente maior nos grupos com maior poder aquisitivo e entre os residentes do Sudeste. Por outro lado, uma vez controlada a análise por idade, sexo e morbidade, as pessoas com planos de saúde apresentam maior chance de uso de serviços de saúde comparativamente às pessoas que não têm planos de saúde. Estes resultados estão em concordância com estudos que apontam o vínculo com planos de saúde como um importante fator na explicação de desigualdades no consumo de serviços de saúde Mueller (1998). Restruturações recentes incorporadas ao setor de seguros privados de saúde nos Estados Unidos têm resultado em maior desigualdade no consumo de serviços de saúde neste país, que se caracteriza por ter o sistema de saúde menos igualitário dentre os países desenvolvidos (Kuttner, 1999).

Os dados disponíveis sobre o comportamento dos seguros privados no Brasil indicam 


\section{Figura 2}

Taxa proporcional de uso de serviços de saúde, padronizada por idade e sexo, por região e tipo de estabelecimento, 1996/7.

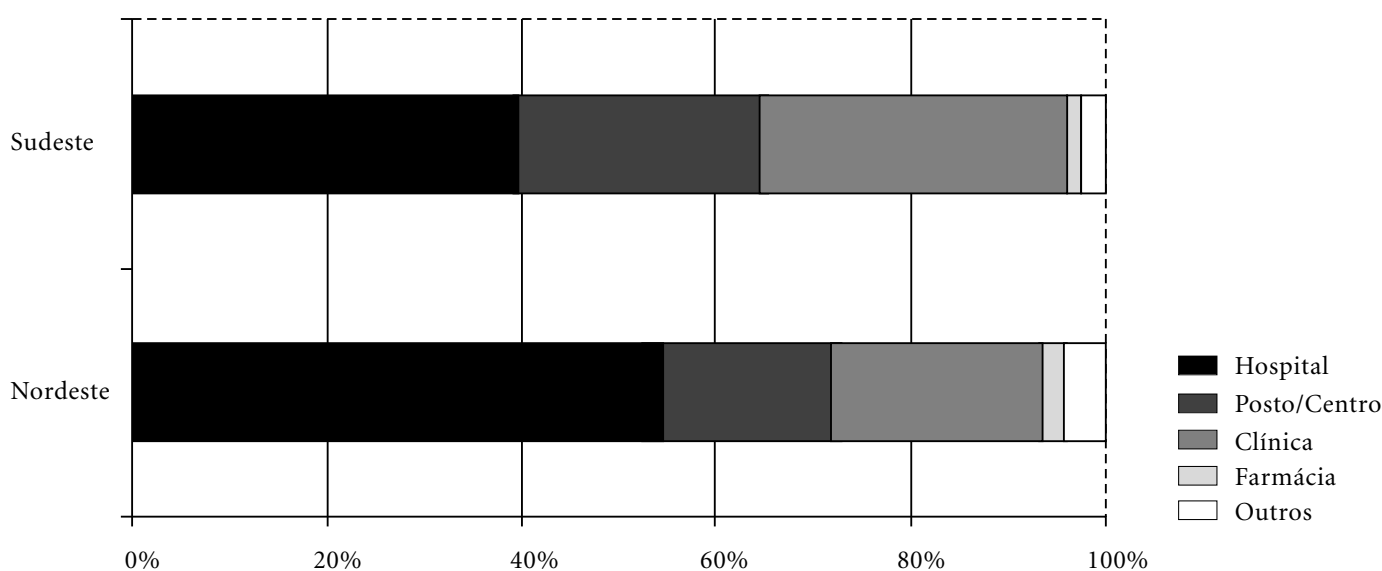

Figura 3

Taxa proporcional de uso de serviços de saúde, por tercil de renda familiar per capita, segundo tipo de estabelecimento.

Nordeste, 1989

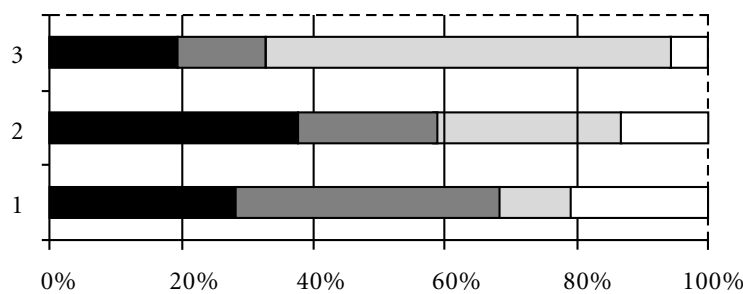

Nordeste, $1996 / 7$

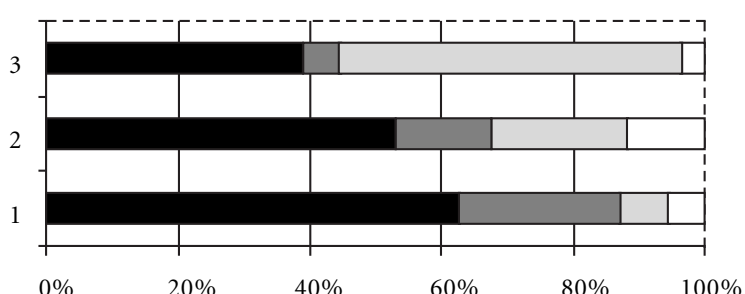

Sudeste, 1989

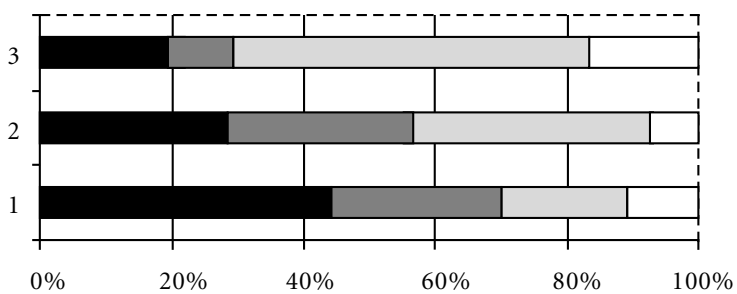

Sudeste, $1996 / 7$

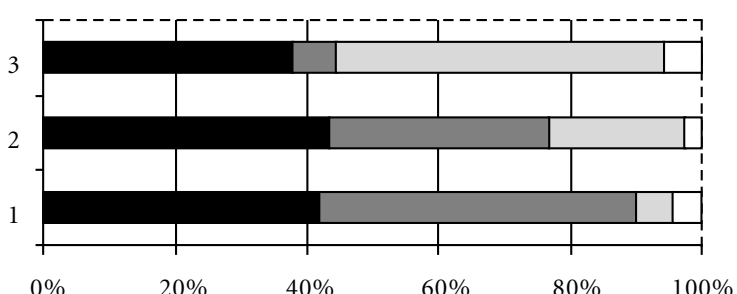

Hospital Clínica

Posto/Centro $\square$ Farmácia/Outro 
igura

Taxa proporcional de uso de serviços de saúde, segundo cobertura por plano de saúde e tipo de serviço utilizado.

Nordeste, 1996/7

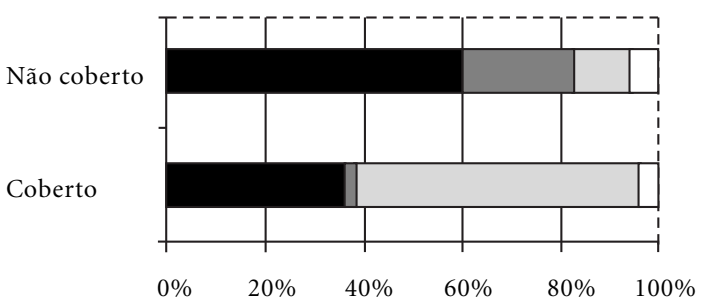

Sudeste, 1996/7

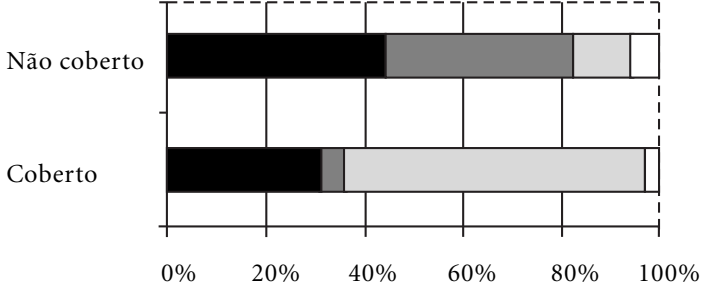

$\begin{array}{ll}\text { Hospital } & \square \text { Clínica } \\ \square \text { Posto/Centro } & \square \text { Farmácia/Outros }\end{array}$ que no período entre 1989 e 1996 houve uma significativa expansão desse subsetor (Almeida, 1998). Essa expansão se dá pari passu à implantação do SUS, contradizendo os princípios da reforma sanitária brasileira, ao acrescentar e acentuar as características seletivas tanto geográficas quanto sociais com relação à cobertura, à utilização e à estrutura de consumo.

A estrutura de consumo de serviços de saúde, medida pela distribuição proporcional do uso segundo o tipo de estabelecimento, modificou-se bastante entre as duas pesquisas, em particular em função de um aumento importante na participação do hospital ${ }^{1}$ na cesta de consumo de serviços de saúde. Este fenômeno ocorreu em ambas as regiões e em todos os grupos de renda, com exceção do primeiro tercil no Sudeste, para o qual a participação dos postos e centros de saúde aumentou substancialmente. $\mathrm{O}$ aumento da participação dos hospitais na estrutura de consumo foi muito mais acentuado no Nordeste e no grupo de mais baixa renda desta região.

A maior participação relativa dos serviços hospitalares é aparentemente paradoxal por alguns motivos. Primeiro, as reformas setoriais das décadas de 1980 e 1990, em geral, objetivaram diminuir os gastos com a assistência médica, o que implica buscar refrear o consumo hospitalar (Abel-Smith \& Mossialos, 1994; Almeida, 1995). Segundo, no Brasil, também foi implantada medida com o objetivo de diminuir o volume de internações hospitalares financiadas pelo SUS (Gouveia et al., 1997), o maior financiador desse tipo de cuidado no país - entre 1993 e 1996 houve redução das taxas de internação financiadas pelo SUS, que foi muito mais acentuada no Sudeste (Almeida, 1998). Terceiro, a cobertura de seguro privado aumentou no período entre as pesquisas (Almeida, 1998), sendo que este setor apresenta, em geral, uma estrutura de consumo pautada por uma baixa participação de serviços hospitalares em relação aos não hospitalares (Ginsburg \& Pickreign, 1996), fato também observado neste estudo, como será exposto mais adiante.

Segundo os dados das pesquisas analisadas neste trabalho, a variação da participação relativa do hospital observada no Nordeste parece refletir um aumento real no uso desse tipo de estabelecimento, pois as taxas de utilização de hospitais nos períodos de referência das pesquisas dobraram entre 1989 e 1996/1997 (3,53 e 7,13 por 100 habitantes respectivamente). Para o Sudeste o aumento verificado foi muito menos importante $(4,59 \mathrm{e}$ 5,58 por 100 habitantes respectivamente).

Considerando o exposto, os resultados observados podem estar indicando que o aumento na participação relativa dos serviços hospitalares se fez, predominantemente, através do aumento do uso de pronto-socorro ou de ambulatório hospitalar, talvez estimulado pela introdução do pagamento por produtividade 
da atenção ambulatorial (Norma Operacional Básica 01/1993), o que significaria uma mudança na estrutura do consumo em uma direção mais ineficiente. Por outro lado, o uso inapropriado de hospitais pode estar relacionado a problemas de acesso (Bindman et al., 1995). Esses resultados podem ser atribuídos às diferenças nos conceitos de tipos de estabelecimento, na formulação das perguntas e nos tempos de referência empregados em cada uma das pesquisas, isto é, o aumento relativo do uso de serviços hospitalares pode ser artefato dos dados utilizados neste estudo. O suplemento saúde da PNAD de 1998 poderá permitir o aprofundamento e a elucidação de algumas destas questões.

Apesar das limitações constatadas, observase consistente variação na estrutura de consumo entre os grupos de renda, o que permite afirmar que a 'cesta' de consumo de cada grupo é distinta nas duas regiões e em ambos os períodos analisados. $\mathrm{O}$ uso de postos e centros de saúde diminui com o aumento da renda e, inversamente, o uso de consultório privado e de clínica aumenta com a renda. Por outro lado, como citado anteriormente, o uso de serviços hospitalares tende a diminuir com o aumento da renda. Os indivíduos cobertos por plano de saúde também apresentam estrutura de consumo diferente dos indivíduos não cobertos. Esta estrutura é semelhante à estrutura do grupo de mais alta renda e caracterizase pela baixa participação relativa do hospital e alta participação de clínica e consultório privado. Este padrão corresponde ao modelo assistencial dominante no subsetor de seguro privado, no qual o uso de serviços hospitalares é mantido sob controle pelas próprias operadoras (Bodenheimer, 1999).

As variações sociais na estrutura de consumo de serviços de saúde, provavelmente, exprimem desigualdades na qualidade do tratamento recebido. Esta hipótese não pôde ser testada, pois dados de inquéritos populacionais são limitados para o estudo de desigualdades na qualidade do tratamento. Porém, alguns dados da PPV permitem abordar a questão do acesso, como por exemplo, os que apontam que os mais pobres esperaram mais do que o dobro do tempo para serem atendidos (82,52 minutos em média para o primeiro tercil de renda e 34,88 para o terceiro tercil). A variação no tempo médio de espera entre os grupos sociais está associada à variação na 'cesta' de consumo destes grupos, na me- dida que esse tempo não é o mesmo em todos os tipos de estabelecimentos: em média cerca de meia hora nas clínicas e consultórios privados e de mais de uma hora nos outros estabelecimentos. O tempo de espera para atendimento das pessoas com seguro privado é, em média, a metade do tempo das pessoas sem seguro.

Como a chance de usar um determinado tipo de estabelecimento é associada à renda do indivíduo, assim como à cobertura por plano de saúde, estabelece-se a reprodução das desigualdades no interior do sistema de saúde, na medida que as possibilidades de escolha no consumo de serviços estão limitadas pelos fatores citados.

A análise do gasto privado com planos de saúde e medicamentos demonstra mais uma dimensão deste intricado processo de reprodução das desigualdades no consumo de serviços de saúde. Apesar de os dados indicarem que o gasto com planos de saúde cresce com a renda, este resultado merece alguma reflexão, pois as diferenças observadas provavelmente estão associadas a contratos distintos, mais restritos nos casos dos que gastam menos. Assim, os mais pobres pagam em média valores inferiores, têm direito a um elenco mais reduzido e menos diversificado de serviços, e o peso desse gasto na renda domiciliar tende a ser excessivamente alto.

Os gastos com medicamentos têm um padrão semelhante ao padrão de gastos com plano de saúde, o que significa que os mais pobres, que têm mais morbidade e que portanto teriam maior necessidade de consumir medicamentos, tendem a gastar menos com este item. A restrição ao consumo de medicamentos entre as pessoas com baixo poder aquisitivo fica evidente quando se verifica que o peso desse gasto na renda domiciliar é proporcionalmente maior do que para as pessoas com renda mais alta.

\section{Conclusão}

A análise aqui apresentada evidencia a enorme complexidade do sistema de saúde brasileiro, constituído por vários mercados de serviços de saúde, estruturados segundo lógicas de oferta e demanda próprias e seletivas, que se imbricam de forma a agravar cumulativamente o quadro de desigualdades no consumo de serviços de saúde. Ao mesmo tempo em que 
se observa um movimento de tímida redução dos níveis de desigualdades no período analisado (1989-1996/1997), a situação atual ainda está longe de atingir uma dinâmica francamente promissora de alcance de maiores graus de eqüidade.

Neste contexto, chama a atenção a insuficiência da produção de dados voltados para o acompanhamento da implementação das políticas, assim como a incipiência de estudos sistemáticos voltados para a avaliação do sistema, dos modelos assistenciais e dos serviços de saúde nesse processo de reforma. Inquéritos populacionais sobre condições de vida e saúde têm sido realizados periodicamente em grande número de países, com maior ou menor freqüência, para orientar formulação das políticas públicas e a alocação de recursos. Nos Estados Unidos, os tradicionais inquéritos de morbidade realizados desde a década de 1960 passaram a ser complementados por inquéri- tos sobre acesso e utilização de serviços de saúde. ${ }^{2}$ Esse mesmo tipo de investigação vem sendo feito no Reino Unido, ${ }^{3}$ Canadá, ${ }^{4}$ Suécia, ${ }^{5}$ Dinamarca $^{6}$ e Jamaica, ${ }^{7}$ para citar alguns exemplos. Na América do Sul, a Colômbia, desde a elaboração da proposta de reforma setorial nos anos 80 , vem realizando vários inquéritos populacionais a fim de constituir uma base de dados para o acompanhamento e avaliação das políticas de saúde em implantação.

A implementação dos princípios do SUS, como um sistema misto pautado na universalização do atendimento à saúde dos cidadãos, inscrita como direito constitucional, depende de uma regulação pública efetiva e da construção de mecanismos capazes de criar maior solidariedade na contribuição financeira necessária à manutenção de um sistema universal, no qual o consumo seja orientado pelas necessidades e não pela capacidade de compra dos indivíduos.

\section{Notas}

* Este trabalho é um dos resultados do projeto A Reforma Sanitária Brasileira: em Busca da Eqüidade, coordenado por Célia Maria de Almeida, financiado pela Organização Pan-Americana da Saúde.

1 Hospital, neste caso, representa não apenas internação, mas também atendimento em pronto-socorro e em ambulatório hospitalar

2 The National Health Interview Survey - conduzido anualmente, foi a campo pela primeira vez em 1973, tendo sido reformulado em 1985 e em 1996.

3 General Household Survey - conduzido anualmente em uma amostra de 20.000 pessoas residentes em domicílios privados que incorpora aspectos relativos ao uso de serviços de saúde e morbidade.

4 A Enquéte Santé Québec vem sendo conduzida desde 1980. O questionário foi reformulado em 1987, 1993 e 1998.

5 The Swedish Survey of Living Conditions, é levada anualmente a campo desde 1974 .

6 No Danish Health Interview Survey Program o levantamento foi a campo em 1987 e 1994.

7 Jamaica Survey of Living Conditions - as informações sobre saúde fazem parte do inquérito realizado em 1989.

\section{Referências bibliográficas}

Abel-Smith B \& Mossialos E 1994. Cost containment and health care reform: a study of the European Union. Health Policy 28 (2):89-132.

Almeida CM 1995. As reformas sanitárias nos anos 80: crise ou transição? Tese de doutorado. Escola Nacional de Saúde Pública, Fundação Oswaldo Cruz, Rio de Janeiro. 460 pp.

Almeida CM 1998. O mercado privado de serviços de saúde no Brasil: panorama atual e tendências da assistência médica suplementar. Texto para discussão no 599. Instituto de Pesquisa Econômica Aplicada (Ipea), Brasília. 81pp.

Almeida CM, Travassos C, Porto S \& Baptista TWF 1999a. A reforma sanitária brasileira: em busca da eqüidade. Research in Public Health, Technical Report, Washington, D.C.: PAHO. 89 pp.

Almeida CM, Travassos C, Porto S \& Labra ME 1999b. Health reform in Brazil: a case on inequity. International Journal of Health Services 30 (1) (no prelo).

Bindman AB et al. 1995. Preventable hospitalizations and access to health care. JAMA 274 (4):305-311.

Black D, Morris JN, Towensend P \& Smith C 1990. The black report, pp. 213. In P Towensend \& N Davidson. Inequalities in Health. Penguin Books, Londres.

Bodenheimer T 1999. The American health care system: the movement for improved quality in health care. New England Journal of Medicine 340 (6):488-492.

Carr-Hill RA 1994. Efficiency and equity implications of the health care reforms. Social Science and Medicine 39 (9):1.189-1.201. 
Castellanos PL 1997. Perfis de mortalidade, nível de desenvolvimento e ineqüidades sociais na região das Américas, pp.137-160. In RB Barata et al. (orgs.). Eqüidade e Saúde: Contribuições da Epidemiologia. Fiocruz/Abrasco, Série Epidemiológica 1, Rio de Janeiro.

Daniels N 1982. Equity of access to health care: some conceptual and ethical issues. Milbank Memorial Fund Quarterly 60 (1):51-81.

Evans 1994. Introduction, pp. 3-26. In RG Evans, ML Batrer \& TR Marmor (eds.). Why Are Some People Healthy and Others Not? The Determinants of Health of Populations. Aldine De Gruyter, Nova York.

Fletcher PR 1988. Pesquisa Nacional Sobre Saúde e Nutrição: a seleção dos elementos da amostra na segunda etapa de amostragem. IBGE. (Mimeo) Documento do IBGE

Ginsburg PB \& Pickreign JD 1996. Tracking health care costs. Health Affairs 15 (3):140-149.

Gouveia CSD, Travassos C \& Fernandes C 1997. Produção de serviços e qualidade da assistência hospitalar no Estado do Rio de Janeiro, Brasil - 1992-95. Revista de Saúde Pública 31(6):601-617.

Hart JT 1971. The inverse care law. The Lancet 1 (7696): 405-412, February 27, 1971.

Hulka B \& Wheat J 1985. Patterns of utilization: a patient perspective. Medical Care 23(5):438-460.

IBGE 1998. Pesquisa sobre Padrões de Vida 1996-1997. Microdados, Rio de Janeiro, em formato CD-ROM

Illsley R \& Baker D 1991. Contextual variations in the meaning of health inequality. Social Science and Medicine 32 (4):359-365.

Illsley R \& Wnuk-Lipinski E 1990. International comparative analysis: main findings and conclusions. Social Science and Medicine 31 (8):879-889.

Kuttner R 1999. The American health care system: employer-sponsored health coverage. New England Journal of Medicine 340 (3):248-252.
Marmot MG 1986. Social inequalities in mortality: the social environment, pp. 21-33. In RG Wilkinson (ed.). Class and Health: Research and Longitudinal Data. Tavistock, Londres.

Mueller KJ, Patil K \& Boilesen E 1998. The role of uninsurance and race in healthcare utilization by rural minorities. Health Services Research 33(3):597610.

O’Donnel O \& Propper C 1989. Equity and the distribution of National Health Service resources. Discussion Paper WSP/45. London School of Economics. Londres. 25pp.

Pessoa GCP \& Silva PLN 1998. Análise de dados amostrais complexos. Associação Brasileira de Estatística, São Paulo. 187 pp.

Porto SM 1997. Eqüidade na Distribuição Geográfica dos Recursos em Saúde: uma Contribuição para o Caso Brasileiro. Tese de doutorado. Escola Nacional de Saúde Pública, Fundação Oswaldo Cruz, Rio de Janeiro.

Shah BV, Barnwell BG \& Bieler GS 1997. SUDAAN User's Manual. Release 7.5, Research Triangle Park, NC.

Travassos C 1992. Equity in the Use of Private Hospitals Contracted by a Compulsory Insurance Scheme in the City of Rio de Janeiro, Brazil, in 1986. Tese de doutorado. The London School of Economics and Political Science, Londres. 282 pp.

Travassos C 1997. Eqüidade e o Sistema Único de Saúde: uma contribuição para o debate. Cadernos de Saúde Pública 2(13):325-330.

Wennberg JE 1985. On patient need, equity, supplierinduced demand and the need to assess the outcome of common medical practices. Medical Care 23(5): 512-520.

Whitehead M 1992. The concepts and principles of equity in health. International Journal of Health Services 22 (3):429-445. 